Pacific Journal of Mathematics

A PROOF OF THE LOWER BOUND CONJECTURE FOR 


\section{A PROOF OF THE LOWER BOUND CONJECTURE FOR CONVEX POLYTOPES}

\section{DAVID BARNETTE}

A $d$-polytope is defined to be a $d$-dimensional set that is the convex hull of a finite number of points. A $d$-polytope is said to be simplicial if each facet is a simplex. Dually, a $d$-polytope is simple if each vertex has valence $d$. It has been conjectured that the following inequalities hold for any simplicial $d$-polytope $p$.

$$
\begin{aligned}
& \text { (1) } f_{k} \geqq\left(\begin{array}{l}
d \\
k
\end{array}\right) f_{0}-\left(\begin{array}{l}
d+1 \\
k+1
\end{array}\right) k \text { for all } 1 \leqq k \leqq d-2 \\
& \text { (2) } f_{d-1} \geqq(d-1) f_{0}-(d+1)(d-2)
\end{aligned}
$$

Here, $f_{i}$ is the number of $i$-dimensional faces of $P$. This conjecture is known as the Lower Bound Conjecture, hereafter to be abbreviated LBC. The LBC has been known to be true for $d \leqq 3$ for quite some time. In 1969 , D. Walkup proved the LBC for $d=4$ and 5 . In 1970, the author proved (2) for all simplicial $d$-polytopes. In this paper $(1)$ is proved for all simplicial $d$-polytopes.

1. Definitions and preliminary results. If $v$ is a vertex of a $d$-polytope $P$ then the antistar of $v$ in $P$, denoted ast $(v, P)$, is the set of all $k$-faces of $P$ that miss $v, 0 \leqq k \leqq d-1$. If $H$ is a hyperplane that separates $v$ from the other vertices of $P$, then $P \cap H$ is called the vertex figure of $v$. If $P$ is simplicial, then the vertex figure of $v$ will be a simplicial $(d-1)$-polytope and each $k$-face of the vertex figure is the intersection of $H$ with a $(k+1)$-face of $P$.

Let $X$ be a collection of facets of a simple $d$-polytope $P$. We say that $X$ is a strong set of facets provided that given any two facets $\mathscr{F}_{1}$ and $\mathscr{F}_{n}$ in $X$ there is a sequence $\mathscr{F}_{1}, \cdots, \mathscr{F}_{n}$ of facets in $X$ such that $\mathscr{F}_{i} \cap \mathscr{F}_{i+1}$ is a subfacet of $P$ for all $1 \leqq i \leqq n-1$. A vertex $v$ of $X$ is said to be an exterior vertex of $X$ provided that $v$ belongs to exactly one facet in $X$.

The graph of a polytope is the graph formed by its vertices and edges. A graph is said to be $n$-connected provided that between any two vertices there are $n$ independent paths (that is, paths that meet only at their endpoints). A theorem of Balinski [1] states that the graph of a $d$-polytope is $d$-connected. The following also follows from Balinski's work.

LEMMA 1. The set of vertices of any facet of a d-polytope does not separate the graph of the polytope. 
2. The main result. We begin with a theorem of M. Perles (private communication).

THEOREM 1. If the LBC is true for edges of simplicial polytopes then it is true for faces of all dimensions.

Proof. For simplicity we shall define $\varphi_{k}(v, d)=\left(\begin{array}{l}d \\ k\end{array}\right) v-\left(\begin{array}{l}d+1 \\ k+1\end{array}\right) k$. We shall prove this theorem for $k$-faces of simplicial $d$-polytopes $2 \leqq k \leqq d-2$. The proof for $k=d-1$ is similar. Our proof is by induction on $d$. It is well known that the $\mathrm{LBC}$ is true for $d=3$. Suppose the LBC is true for edges of simplicial polytopes of all dimensions and for $k$-faces $2 \leqq k \leqq d-3$ for simplicial $(d-1)$-polytopes. Let $P$ be a simplicial $d$-polytope with $v$ vertices, $p_{1}, \cdots, p_{v}$. Let $v_{i}$ be the valence of $p_{i}$.

By induction, the number of $(k-1)$-faces in the vertex figure of $p_{i}$ is at least $\varphi_{k-1}\left(v_{i}, d-1\right)$ thus $p_{i}$ is incident to at least $\varphi_{k-1}\left(v_{i}, d-1\right)$ $k$-faces of $P$.

The number of incidences of vertices and $k$-faces in $P$ is at least

$$
\begin{aligned}
\sum_{i=1}^{v} \varphi_{k-1}\left(v_{i}, d-1\right) & \geqq \sum_{i=1}^{v}\left[\left(\begin{array}{l}
d-1 \\
k-1
\end{array}\right) v_{i}-\left(\begin{array}{l}
d \\
k
\end{array}\right)(k-1)\right] \\
& \geqq-v\left(\begin{array}{l}
d \\
k
\end{array}\right)(k-1)+\left(\begin{array}{l}
d-1 \\
k-1
\end{array}\right) \sum_{i=1}^{v} v_{i} .
\end{aligned}
$$

But

$$
\sum_{i=1}^{v} v_{i}=2 E
$$

By the LBC for edges

$$
\sum_{i=1}^{v} v_{i} \geqq 2 d v-d^{2}-d .
$$

Thus using (3) and (4) we have

$$
\begin{aligned}
\sum \varphi_{k-1}\left(v_{i}, d-1\right) & \geqq\left(\begin{array}{l}
d-1 \\
k-1
\end{array}\right)\left[2 d v-d^{2}-d\right]-v\left(\begin{array}{l}
d \\
k
\end{array}\right)(k-1) \\
& \geqq 2 k\left(\begin{array}{l}
d \\
k
\end{array}\right) v-k\left(\begin{array}{l}
d \\
k
\end{array}\right)+\left(\begin{array}{l}
d \\
k
\end{array}\right) v-\left(\begin{array}{l}
d+1 \\
k+1
\end{array}\right) k(k+1) \\
& \geqq\left(\begin{array}{l}
d \\
k
\end{array}\right) v(k+1)-\left(\begin{array}{l}
d+1 \\
k+1
\end{array}\right) k(k+1) .
\end{aligned}
$$

We also know that the number of incidences of vertices and $k$-faces is $(k+1) f_{k}$, thus 


$$
(k+1) f_{k} \geqq\left(\begin{array}{l}
d \\
k
\end{array}\right) v(k+1)-\left(\begin{array}{l}
d+1 \\
k+1
\end{array}\right) k(k+1)
$$

and the lower bound inequality follows.

We shall also use the following:

LEMMA 2. If $f_{1} \geqq d v-K$ holds for all simplicial d-polytopes, where $K$ is a constant depending only on $d$, then the $\mathrm{LBC}$ is true for edges of simplicial d-polytopes.

Proof. Suppose $P$ is a simplicial $d$-polytope for which the LBC does not hold for edges. Then we have

$$
f_{1}=d v-\frac{d^{2}+d}{2}-r
$$

for some positive integer $r$.

Let $\mathscr{F}$ be a facet of $P$ and let $H$ be the affine hull of $\mathscr{F}$. Let $P^{\prime}$ be the union of $P$ and the reflection $P$ through $H$. The set $P^{\prime}$ may not be convex, however, if a suitable projective transformation is applied to $P$ first, then $P^{\prime}$ will be a simplicial $d$-polytope. The number of edges of $P^{\prime}$ is

$$
2 d v-d^{2}-d-2 r-\frac{d^{2}-d}{2}=(2 v-d) d-\frac{d^{2}+d}{2}-2 r .
$$

But $2 v-d$ is the number of vertices of $X$, thus $P^{\prime}$ fails to satisfy the $\mathrm{LBC}$ by twice as many edges as does $P$. By reflecting $P^{\prime}$ about the affine hull of a facet we can get a simplicial $d$-polytope $P^{\prime \prime}$ that fails to satisfy the LBC by at least $4 r$ edges. Continuing this process eventually produces a polytope for which $f_{1}<d v-K$ which is a contradiction.

In proving the LBC we shall work in the duals of simplicial polytopes - the simple $d$-polytopes. We will need the following lemma about the boundaries of simple $d$-polytypes.

LEMma 3. Let $X$ be a strong set of facets in the boundary of a simple d-polytype $P$ such that $X$ misses at least one vertex of $P$. Then there is an exterior vertex of $v$ of $X$ belonging to a facet $\mathscr{F}$ of $X$ such that ast $(v, \mathscr{F})$ separates $X$ into exactly two strong components (one of which is the facet $\mathscr{F}$ ).

Proof. Since the graph of $P$ is connected there is a path from $X$ to some vertex of $P$ not in $X$. The first edge of this path belongs to $d-1$ facets of $P$ that are not in $X$, while one vertex $v$ of the edge is in $X$ thus $v$ is an exterior vertex of $X$. 
Among all exterior vertices of $X$ choose $v$ such that some component $\mathscr{C}$ of $X \sim$ ast $(v, \mathscr{F})$ has a maximum number of facets. Suppose there is some component $\mathscr{C}_{1}$ of $X \sim$ ast $(v, \mathscr{F})$ other than the maximal component and $\mathscr{F}$. If $\mathscr{C}_{1}$ has no exterior vertices other than those on $\mathscr{F}$ then we could separate the graph of $P$ by removing the vertices of $\mathscr{F}$ which contradicts Lemma 1 . Thus $\mathscr{C}$ has an exterior vertex $v_{1}$ not on $\mathscr{F}$. This implies that $v_{1}$ is an exterior vertex of $X$. Let $v_{2} \in \mathscr{F}_{1}$ such that $\mathscr{F}_{1}$ is a facet in $X$. Now we reach a contradiction because $\mathscr{C} \cup \mathscr{F}$ determines a component of $X \sim \operatorname{ast}\left(v_{1}, \mathscr{F}_{1}\right)$ with more facets than $\mathscr{C}$. We are now ready to prove our main theorem.

THEOREM 2. If $P$ is a simple d-polytope with $F$ facets and $S$ subfacets then $S \geqq d F-d^{2}-d$.

Proof. Let $v$ be a vertex of $P$ and let $X=\operatorname{ast}(v, P)$. Let $v_{1}$ be an exterior vertex of $X$ such that separating $X$ by removing ast $\left(v_{1}, \mathscr{F}_{1}\right)$ yields two components, $\left(\mathscr{F}_{1}\right.$ is the facet of $X$ containing $\left.v_{1}\right)$. Let $X_{1}$ be the closure of the component that misses $\mathscr{F}$ and let $\mathscr{S}_{1}=X_{1} \cap \mathscr{F}_{1}$. We shall call $\mathscr{S}_{1}$ a separating set. In $X_{1}$ we choose an exterior vertex $v_{2}$ belonging to a facet $\mathscr{F}_{2}$ of $X_{1}$, such that ast $\left(v_{2}, \mathscr{F}_{2}\right)$ separates $X_{1}$ into two components one of which is $\mathscr{F}_{2}$. The closure of the component that misses $\mathscr{F}_{2}$ we shall call $X_{2}$. We let $\mathscr{S}_{2}$ be the separating set $\mathscr{F}_{2} \cap X_{2}$. Continuing in this way we get a sequence $X, X_{1}, \cdots, X_{n}$ of strong sets of facets, a sequence of separating sets $S_{1}, \cdots, S_{n}$, and a sequence $\mathscr{F}_{1}, \cdots, \mathscr{F}_{n}$ of facets in $X, n=F-d-1$.

Consider any facet $\mathscr{F}_{1}$ in the sequence $\mathscr{F}_{1}, \cdots, \mathscr{F}_{n}$. Let $v_{1}^{\prime}, \cdots, v_{k}^{\prime}$ be the vertices in the dual $\mathscr{F}_{i}^{*}$ of $\mathscr{F}_{i}$, corresponding to the facets of $\mathscr{F}_{1}$ in $\mathscr{S}_{i}$. Let $\omega$ be a point that is joined to each vertex of the facet $f$ of $\mathscr{F}_{i}^{*}$ corresponding to $v_{i}$ in $\mathscr{F}_{i}$. Thus, the graph that is the union of the graph of $\mathscr{F}_{i}^{*}, \omega$ and the edges from $\omega$ to $f$ is the graph of the $(d-1)$-polytope obtained by taking the convex hull of $\mathscr{F}_{i}$ and a point near the centroid of $f$. There exist $d-1$ independent paths $\Gamma_{1}, \cdots, \Gamma_{d-1}$ from $\omega$ to $v_{1}^{\prime}$. On each path $\Gamma_{i}$ let $v_{i}^{\prime \prime}$ be the last vertex encountered before first encountering any vertex in $\left\{v_{1}^{\prime}, \cdots, v_{n}^{\prime}\right\}$ as we traverse $\Gamma_{i}$ from $\omega$ to $v_{1}^{\prime}$. The vertices $v_{i}^{\prime \prime}$ are distinct and correspond to $d-1$ subfacets of $P$ that lie on $\mathscr{F}_{i}$ and meet $\mathscr{S}_{i}$ on $(d-3)$-faces of $P$.

This gives us $d-1$ subfacets on each of the facets $\mathscr{F}_{1}, \cdots, \mathscr{F}_{n}$. This gives us at least $(d-1)(F-d-1)$ subfacets in $P$. These subfacets will be called facial subfacets.

Next we show that we can find some subfacets in the separating sets that are not facial subfacets. Clearly there are many subfacets 
on the separating sets; however, in choosing a subfacet on a separating set $\mathscr{S}_{i}$ we must be sure that later on it is not a facial subfacet on some $\mathscr{F}_{j}, j>i$. A subfacet in a separating set $\mathscr{S}_{i}$ will be a facial subfacet only if it meets some other separating set on a $(d-3)$-face. Thus we must consider separating sets that meet $\mathscr{S}_{i}$ on a $(d-3)$-face. Furthermore, we need only worry about such separating sets $\mathscr{S}_{j}$ for $j>i$.

Suppose $\mathscr{S}_{j}$ meets $\mathscr{S}_{i}$ on a $(d-3)$-face $H$. The $(d-3)$-face $H$ belongs to exactly three facets, $\mathscr{F}_{i}, \mathscr{F}_{j}$ and one other facet which we shall call $\mathscr{F}_{k}$, and to three subfacets. This implies that $\mathscr{F}_{k} \cap \mathscr{F}_{i}$ is not empty and thus is a subfacet. Thus when $\mathscr{S}_{j}$ separates $X_{j}$ it also separates $\mathscr{S}_{i}$, and we may choose a component $\mathscr{C}$ of this separated separating set that contains a subfacet that does not become a facial subfacet when we remove $\mathscr{F}_{j}$.

We need to show that we can continue in this way, that is, if some other separating set $\mathscr{S}_{l}$ meets $\mathscr{C}$ then $\mathscr{C}$ is separated by $\mathscr{S}_{l}$ (not merely that $\mathscr{S}_{i}$ is separated by $\mathscr{S}_{l}$ ). As in the above argument if $\mathscr{S}_{l}$ meets $\mathscr{C}$ then there are three facets $\mathscr{F}_{i}, \mathscr{F}_{l}$ and a third facet $\mathscr{F}_{m}$ meeting on a $(d-3)$-face $H^{\prime}$ of $\mathscr{C}$. It is clear that $\mathscr{F}_{m} \cap \mathscr{F}_{i}$ is a subfacet in $\mathscr{S}_{i}$. If it is not also a subfacet in $\mathscr{C}$, then we have that the subfacet $\mathscr{F}_{i} \cap \mathscr{F}_{l}$ in $\mathscr{C}$ meets a subfacet $\mathscr{F}_{i} \cap \mathscr{F}_{m}$ not in $\mathscr{C}$, and thus $H^{\prime}$ would belong to the previous separating set meeting $\mathscr{S}_{i}$, namely $\mathscr{S}_{j}$. Since clearly the separating sets are subfacet disjoint we now have at least four subfacets containing $H^{\prime}$, two on $\mathscr{S}_{i}$ and one each on $\mathscr{S}_{j}$ and $\mathscr{S}_{l}$, which is a contradiction.

Since $\mathscr{F}_{i} \cap \mathscr{F}_{m}$ is in $\mathscr{C}$ we see that $\mathscr{S}_{n}$ separates $\mathscr{C}$ and at least one component contains a subfacet that does not become a facial subfacet when we remove $\mathscr{F}_{n}$. With repetition of the above argument we may eventually arrive at a subfacet in $\mathscr{S}_{i}$ that is not a facial subfacet; we now choose one such subfacet from each separating subset.

Now, we have found $(d-1)(F-d-1)+F-d-1$ subfacets, thus $S \geqq d F-d^{2}-d$.

THEOREM 3. If $P$ is a simplicial d-polytope with $f_{k}$-faces then

$$
f_{k} \geqq\left(\begin{array}{l}
d \\
k
\end{array}\right) f_{0}-\left(\begin{array}{l}
d+1 \\
k+1
\end{array}\right) k \text { for all } 1 \leqq k \leqq d-2 \text {. }
$$

Proof. The proof follows by taking the dual of Theorem 3 applying Lemma 2 and then applying Theorem 1.

3. Remarks. 1. The proof of the LBC for facets in [2] can be simplified by using Lemma 3 . 
2. Using some unpublished theorems of Walkup dealing with graph of more general structures one can prove that the lower bound inequalities hold for all triangulated manifolds. See also [3] for similar results.

3. Let $P$ be a simplicial polytope obtained by starting with the $d$-simplex and repeatedly adding simplicial caps to the facets. Such a polytope is called a stacked polytope. It has been conjectured that equality in (1) or (2) holds only for stacked polytopes. In [2] it is shown to be true for (1) but for inequality (2) the question is still open.

4. Theorem 1 seems to be a part of the folklore of convex polytopes. To the best of the author's knowledge it was first proved by M. Perles in 1966 and communicated to G. T. Sallee by telephone. The author is indebted to Professor Sallee for his accurate note taking which has enabled the author to reconstruct Perles' proof.

\section{REFERENCES}

1. M. Balinski, On the graph structure of convex polyhedra in n-space, Pacific J. Math., 11 (1961), 431-434.

2. D. Barnette, The minimum number of vertices of a simple polytope, Israel J. of Math., 10 (1971), 121-125.

3. - Graph theorems for manifolds, to appear, Israel J. Math.

4. B. Grünbaum, Convex Polytopes, Wiley and Sons, New York, 1967.

5. D. Walkup, The lower bound conjecture for 3- and 4-manifolds, Acta Mathematica, 125 (1970), 75-107.

Received April 6, 1972. Research supported by NSF Grant GP-27963.

University of CALIFornia, Davis 


\section{PACIFIC JOURNAL OF MATHEMATICS}

\section{EDITORS}

D. Gilbarg and J. Milgram

Stanford University

Stanford, California 94305

\author{
R. A. Beaumont \\ University of Washington \\ Seattle, Washington 98105
}

J. DuGUNDJI

Department of Mathematics

University of Southern California

Los Angeles, California 90007

Richard ARENS

University of California

Los Angeles, California 90024

\section{ASSOCIATE EDITORS}
E. F. BECKENBACH
B. H. NeumanN
F. WOLF
K. YosHIDA

\section{SUPPORTING INSTITUTIONS}

UNIVERSITY OF BRITISH COLUMBIA

CALIFORNIA INSTITUTE OF TECHNOLOGY

UNIVERSITY OF CALIFORNIA

MONTANA STATE UNIVERSITY

UNIVERSITY OF NEVADA

NEW MEXICO STATE UNIVERSITY

OREGON STATE UNIVERSITY

UNIVERSITY OF OREGON

OSAKA UNIVERSITY

\author{
UNIVERSITY OF SOUTHERN CALIFORNIA \\ STANFORD UNIVERSITY \\ UNIVERSITY OF TOKYO \\ UNIVERSITY OF UTAH \\ WASHINGTON STATE UNIVERSITY \\ UNIVERSITY OF WASHINGTON
* * * *
AMERICAN MATHEMATICAL SOCIETY \\ NAVAL WEAPONS CENTER
}

The Supporting Institutions listed above contribute to the cost of publication of this Journal, but they are not owners or publishers and have no responsibility for its content or policies.

Mathematical papers intended for publication in the Pacific Journal of Mathematics should be in typed form or offset-reproduced, (not dittoed), double spaced with large margins. Underline Greek letters in red, German in green, and script in blue. The first paragraph or two must be capable of being used separately as a synopsis of the entire paper. The editorial "we" must not be used in the synopsis, and items of the bibliography should not be cited there unless absolutely necessary, in which case they must be identified by author and Journal, rather than by item number. Manuscripts, in duplicate if possible, may be sent to any one of the four editors. Please classify according to the scheme of Math. Rev. Index to Vol. 39. All other communications to the editors should be addressed to the managing editor, Richard Arens, University of California, Los Angeles, California, 90024.

50 reprints are provided free for each article; additional copies may be obtained at cost in multiples of 50 .

The Pacific Journal of Mathematics is issued monthly as of January 1966. Regular subscription rate: $\$ 48.00$ a year (6 Vols., 12 issues). Special rate: $\$ 24.00$ a year to individual members of supporting institutions.

Subscriptions, orders for back numbers, and changes of address should be sent to Pacific Journal of Mathematics, 103 Highland Boulevard, Berkeley, California, 94708.

PUBLISHED BY PACIFIC JOURNAL OF MATHEMATICS, A NON-PROFIT CORPORATION

Printed at Kokusai Bunken Insatsusha (International Academic Printing Co., Ltd.), 270, 3-chome Totsuka-cho, Shinjuku-ku, Tokyo 160, Japan. 


\section{Pacific Journal of Mathematics}

\section{Vol. 46, No. 2 December, 1973}

Christopher Allday, Rational Whitehead products and a spectral sequence of

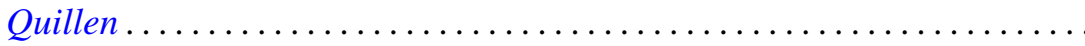

James Edward Arnold, Jr., Attaching Hurewicz fibrations with fiber

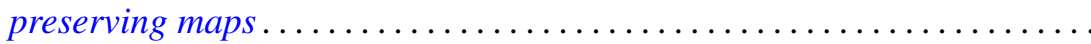

Catherine Bandle and Moshe Marcus, Radial averaging transformations with various metrics.................................

David Wilmot Barnette, A proof of the lower bound conjecture for convex

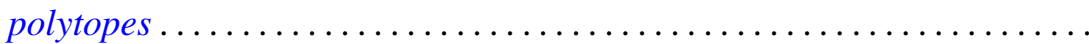

Louis Harvey Blake, Simple extensions of measures and the preservation of

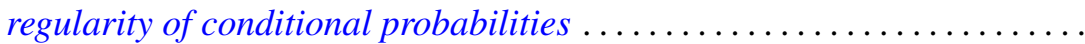

James W. Cannon, New proofs of Bing's approximation theorems for

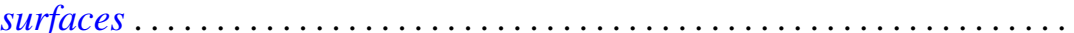

C. D. Feustel and Robert John Gregorac, On realizing HNN groups in

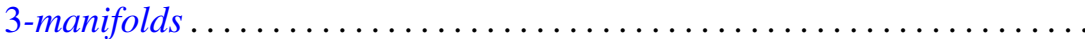

Theodore William Gamelin, Iversen's theorem and fiber algebras . . . . . . . . 389

Daniel H. Gottlieb, The total space of universal fibrations . . . . . . . . . . . .

Yoshimitsu Hasegawa, Integrability theorems for power series expansions of

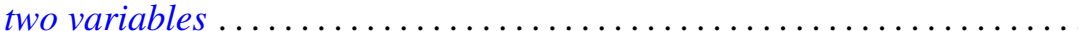

Dean Robert Hickerson, Length of period simple continued fraction expansion of $\sqrt{ } d$

Herbert Meyer Kamowitz, The spectra of endomorphisms of the disc algebra.

Dong S. Kim, Boundedly holomorphic convex domains

Daniel Ralph Lewis, Integral operators on $\mathscr{L}_{p}$-spaces ...

John Eldon Mack, Fields of topological spaces . . . . . . . . .

V. B. Moscatelli, On a problem of completion in bornology

Ellen Elizabeth Reed, Proximity convergence structures. .

Ronald C. Rosier, Dual spaces of certain vector sequence spaces .

Robert A. Rubin, Absolutely torsion-free rings

Leo Sario and Cecilia Wang, Radial quasiharmonic functions . .

James Henry Schmerl, Peano models with many generic classes .

H. J. Schmidt, The $\mathscr{F}$-depth of an $\mathscr{F}$-projector ............

Edward Silverman, Strong quasi-convexity. . . . . . . . . . . . . . . . . 549

Barry Simon, Uniform crossnorms ....................... 555

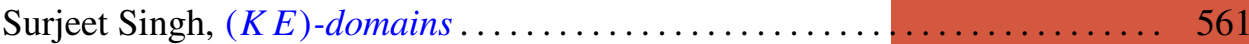

Ted Joe Suffridge, Starlike and convex maps in Banach spaces . . . . . . . . 575

Milton Don Ulmer, $C$-embedded $\Sigma$-spaces . . . . . . . . . . . . . . . . 591

Wolmer Vasconcelos, Conductor, projectivity and injectivity . . . . . . . . . 603 